\title{
Immediate Pain Response Does Not Predict Long-Term Outcome of CT-Guided Cervical Transforaminal Epidural Steroid Injections
}

\author{
J.T. Wald, T.P. Maus, J.R. Geske, F.E. Diehn, T.J. Kaufmann, N.S. Murthy, K.R. Thielen, and S. Watson
}

\begin{abstract}
BACKGROUND AND PURPOSE: Imaging-guided cervical transforaminal epidural steroid injections have been shown to decrease verbal numerical pain scores and improve functionality (Roland Morris Disability Index). These injections are often administered in combination with local anesthetic. The purpose of this study was to determine if the immediate postprocedure VNPS predicts the long-term effectiveness of the injection.
\end{abstract}

MATERIALS AND METHODS: A quality assurance data base review of 247 patient records was used to document the VNPS and RMDI of patients undergoing a single CT-guided CTESI. Pain scores were recorded before the procedure, immediately after the procedure, at 2 weeks, and at 2 months. The RMDI was recorded before the procedure, at 2 weeks, and at 2 months. Spearman rank correlation analysis and logistic regression models were used to determine if the immediate postprocedure or 2-week VNPS correlated with or predicted the longer-term VNPS and RMDI as measured at 2 weeks and 2 months.

RESULTS: There was not a strong correlation between the pain score obtained immediately after the procedure and the 2-month outcome of the VNPS or RMDI. The pain scores at 2 weeks did correlate with the 2-month outcomes. The 2-week VNPS also was a significant predictor of patients who would achieve a $>50 \%$ improvement in VNPS or RMDI at 2 months.

CONCLUSIONS: Pain scores obtained immediately after completion of a single CT-guided CTESI do not predict the long-term effectiveness of this procedure. However, patient response at 2 weeks does correlate with the long-term effectiveness of these injections as measured by the VNPS and the RMDI.

ABBREVIATIONS: CTESI = cervical transforaminal epidural steroid injection; PLA2 = phospholipase A2; RMDI = Roland Morris Disability Index; VNPS = verbal numerical pain score

$\mathrm{t}$

maging-guided cervical transforaminal epidural steroid injections have been shown to decrease verbal numerical pain scores $^{1-5}$ and improve functionality as measured by the Roland Morris Disability Index. ${ }^{6}$ These injections are often administered in combination with local anesthetic to establish whether the patient gets immediate pain relief, suggestive that the correct site is being targeted for the steroid injection. Pain response to local anesthetic has been shown to be a moderate predictor of surgical outcomes. ${ }^{7}$ If local nerve blocks have the potential to predict surgical outcomes, would immediate response to the local anesthetic also predict outcomes for transforaminal epidural injections? The purpose of this retrospective study was to determine if the VNPS reported immediately after a single injection of anesthetic and

Received September 11, 2012; accepted after revision October 20

From the Department of Radiology, Mayo Clinic, Rochester, Minnesota.

Please address correspondence to John T. Wald, MD, Department of Radiology, Mayo Clinic, 200 First St SW, Rochester, MN 55905; e-mail: wald.john2@mayo.edu

http://dx.doi.org/10.3174/ajnr.A3439 steroid predicts the long-term effectiveness of the steroid injection as measured by the VNPS and RMDI at 2 weeks and 2 months.

\section{MATERIALS AND METHODS}

The study was completed at a tertiary referral center in the upper Midwest after institutional review board approval. A retrospective review of a quality assurance data base was completed over a 4 -year period for all patients $(n=247)$ who received CT-guided CTESI. All patients receiving epidural injections completed a preprocedure quality assurance form that included patient demographics, RMDI, ${ }^{6}$ and VNPS: 0 indicates no pain; 10 , worst pain of life. The VNPS was also defined immediately after the procedure in person and at 2 weeks and 2 months after the procedure by telephone interview. RMDI was defined at 2 weeks and 2 months after the procedure by a telephone interview. The 2-week and 2-month follow-up phone calls are performed by an independent assessor employed by the radiology department (not the physician) who enters the data directly into an SAS (SAS Institute, 
Table 1: Demographics, VNPS, and RMDI outcomes

\begin{tabular}{lcc}
\hline \multicolumn{1}{c}{ Group } & No. & Mean \pm SD \\
\hline Age (years) & 247 & $54.0 \pm 12.1$ \\
Male & 144 & $58.3(\%)$ \\
VNPS before & 219 & $5.3 \pm 2.3$ \\
VNPS immediate & 246 & $1.7 \pm 1.9$ \\
VNPS 2 weeks after & 172 & $3.3 \pm 2.5$ \\
VNPS 2 months after & 120 & $2.9 \pm 2.3$ \\
RMDI before & 219 & $9.3 \pm 5.2$ \\
RMDI 2 weeks after & 172 & $6.8 \pm 4.2$ \\
RMDI 2 months after & 120 & $5.0 \pm 3.4$ \\
\hline
\end{tabular}

Cary, NC) quality assurance data base maintained by the Department of Biomedical Statistics and Informatics.

Patients with unilateral radicular pain who underwent a single CT-guided CTESI were included regardless of level of cervical radiculopathy or duration of pain. Patients who received multiple injections within a 2 -month period of time were excluded. The site of injection was determined by the referring service on the basis of diverse evaluation criteria to include imaging findings, clinical examination, electromyography, and so forth. Previous analysis of VNPS and RMDI score of patients with injections with the use of an anterolateral or a posterior approach have shown no difference in outcomes ${ }^{5}$; thus, results from all patients who fit the criteria were included (Tables 1 and 2).

\section{Procedure}

Before the procedure was performed, clinical informed consent was received from all patients. All injections were completed by experienced radiologists trained in CT-guided spine interventions. Physicians were fellowship-trained in neuroradiology or musculoskeletal radiology. Staff experience ranged from 2-25 years, with an average of approximately 10 years of staff experience in CT-guided procedures. Diagnostic CT images were obtained to localize the treatment level by use of standard CT technique. With the use of CT guidance, a 25-ga spinal needle was placed to the lateral margin of the cervical foramen, by use of an anterolateral or a posterior approach. ${ }^{5}$ After confirmation of periganglionic, extradural, and extravascular spread of contrast, 1 $\mathrm{mL}(20 \mathrm{mg}$ ) of lidocaine was infused. After a 2-minute observation period during which neurologic status was assessed, $1 \mathrm{~mL}$ (10 $\mathrm{mg}$ ) of dexamethasone was infused. Patients were monitored for approximately 30-45 minutes after each procedure and then discharged.

\section{Statistical Analysis}

Analyses were conducted to test for association of the patients' immediate postprocedure VNPS with their follow-up VNPS and RMDI at 2 weeks and 2 months by use of Spearman rank correlations. For comparison, correlations also were used to examine the association of 2-week VNPS with 2-month VNPS and RMDI.

Univariate logistic regression models were used to determine whether the immediate, 2 -week, or change from baseline to immediate VNPS were predictors of a $>50 \%$ improvement in the VNPS or RMDI score at 2 weeks or 2 months. This method was also used to determine whether the 2-week RMDI or VNPS predicted a $>50 \%$ improvement in RMDI or VNPS at 2 months. The outcomes, percentage of change in VNPS and
RMDI, were calculated from before the procedure to 2 weeks and from before the procedure to 2 months. Confounders such as baseline pain and loss to follow-up were considered and reported by comparing mean baseline VNPS/RMDI among those $50 \%$ responders and nonresponders and by loss to follow-up status by use of Wilcoxon rank sum tests. Statistical analyses were conducted by use of SAS, Version 9.2 (SAS Institute).

\section{RESULTS}

Two hundred forty-seven patients who underwent a single CTESI were included in this retrospective study, as presented in Tables 1 and 2. The 2-week and 2-month outcomes of these patients have been previously reported. ${ }^{5}$ Preprocedural data were not available for 18 patients $(11 \%)$, and phone interview follow-up data were unavailable for 75 patients $(30 \%)$ at 2-week follow-up and for 127 patients $(51 \%)$ at 2 -month follow-up. There were no differences in mean baseline VNPS by 2-week or 2-month VNPS outcome status (2-week $P=0.794$; 2-month $P=0.246$ ). Mean baseline RMDI was higher in subjects who achieved 50\% improvement at 2 weeks and 2 months than in those who did not achieve this (mean difference \pm SD: 2 weeks, $2.6 \pm 5.2, P=.001 ; 2$ months, $3.7 \pm 4.9, P<.001$ ).

Previously reported data demonstrated a statistically significant decrease in patient pain levels and improvement in functional status as measured by the VNPS and the RMDI between preprocedure scores and the 2-week and 2-month follow-up times. ${ }^{5}$ VNPS recorded immediately after completion of the procedure weakly correlated with 2-week $(\rho=0.264)$ and 2-month $(\rho=0.205)$ outcomes for VNPS and was not clinically useful for predicting pain outcomes (Table 3). Immediate VNPS was weakly correlated with the 2-week RMDI and did not correlate with the 2-month RMDI. In contrast, 2-week VNPS demonstrated a correlation with the 2-month VNPS outcomes and the 2-week $(\rho=0.574, P<.001)$ and 2 -month RMDI outcomes $(\rho=0.514, P<.001)$ (Table 3$)$.

Logistic regression models with immediate or 2-week scores to predict a $50 \%$ or better improvement at later time points led to results similar to the correlation analysis (Table 4). The immediate postprocedure VNPS did not significantly predict an outcome of $>50 \%$ improvement in VNPS or RMDI at 2 weeks or at 2 months. In contrast, the 2-week VNPS did significantly predict the outcome of $>50 \%$ improvement in VNPS at 2 months. Higher 2-week VNPS was significantly associated with a decreased probability of achieving $50 \%$ or better improvement in VNPS from preprocedure VNPS (odds ratio $=0.789, P=.008$ ). The 2-week RMDI score also significantly predicted the outcome of $>50 \%$ improvement in the RMDI at 2 months. Similarly, higher RMDI scores at 2 weeks were significantly associated with a decreased probably of achieving $50 \%$ or better improvement on the RMDI (odds ratio $=0.853, P=.006$ ). Results were similar when adjusting models for baseline VNPS or RMDI. Change in VNPS or RMDI from baseline to immediately after the procedure was not a significant predictor of VNPS or RMDI outcomes at 2 weeks or 2 months.

\section{DISCUSSION}

Image-guided CTESI has been demonstrated to significantly reduce pain and improve function in patients with radicular pain syndrome. ${ }^{1,3-5,8}$ Patients are typically assessed immediately after epidural injections whether the procedure is completed by means of 
Table 2: Proportion of patients at each follow-up visit by qualitative response

\begin{tabular}{lccccc}
\hline & \multicolumn{2}{c}{ VNPS } & & \multicolumn{2}{c}{ RMDI } \\
\cline { 2 - 3 } Response Definition & 2 Weeks & 2 Months & & 2 Weeks & 2 Months \\
\hline All patients & $n=171$ & $n=119$ & & $n=171$ & $n=119$ \\
$>50 \%$ Improvement & $35.1 \%$ & $39.5 \%$ & & $22.8 \%$ & $35.3 \%$ \\
Complete relief & $16.9 \%$ & $17.5 \%$ & & - & - \\
No improvement/worsening & $30.4 \%$ & $29.4 \%$ & & $36.8 \%$ & $32.8 \%$ \\
\hline
\end{tabular}

Note:-Data from Wald et al., AJNR 2012;33:415-19.

Table 3: Spearman rank correlations of VNPS immediately and 2 weeks after injection with VNPS and RMDI 2 weeks and 2 months after injection

\begin{tabular}{lcccc}
\hline $\begin{array}{c}\text { Postprocedure Score } \\
\text { VNPS immediate }\end{array}$ & $\begin{array}{c}\text { VNPS } \\
\text { 2 Weeks }\end{array}$ & $\begin{array}{c}\text { VNPS } \\
\text { 2 Months }\end{array}$ & $\begin{array}{c}\text { RMDI } \\
\text { 2 Weeks }\end{array}$ & $\begin{array}{c}\text { RMDI } \\
\text { 2 Months }\end{array}$ \\
\hline$\rho$ & & & & \\
$P$ & 0.264 & 0.205 & 0.208 & 0.087 \\
VNPS 2 weeks & .001 & .025 & .006 & .347 \\
$\rho$ & & & & \\
$P$ & 1 & 0.574 & 0.676 & 0.514 \\
& - & $<.001$ & $<.001$ & $<.001$ \\
\hline
\end{tabular}

should be cautioned against making predictions of patient outcomes based on the immediate response to the cervical injection. The patient result observed after 2 weeks is a much stronger predictor of the longer-term outcome at 2 months for pain response and functional improvement. The odds ratio of 0.853 indicates that on average, a patient would have 1.9 times better odds of achieving the $50 \%$ improvement than a patient with a 4-point higher baseline RMDI.

The poor correlation between the immediate postprocedure pain score and the long-term outcomes is not necessarily surprising. Though the $2 \%$ lidocaine used in this study may be able to temporarily decrease the cervical radic-

the CT-guided or fluoroscopic-guided approach. As clinicians, we often encourage our patients that improvement immediately after the procedure brings hope of long-term relief. However, there is little literature to support this contention, and, theoretically, the only effect immediately after the procedure should be the anesthetic effect. A significant decrease in the index pain after the procedure may be diagnostic, indicative that the appropriate level has been treated, and it may be predictive of surgical outcomes. ${ }^{7}$ However, it does not necessarily predict a long-term pain response or functional improvement after corticosteroid injection.

Though this study reaffirmed results of previously published data that CT-guided CTESI is effective, the results also demonstrated that immediate postprocedure pain response did not correlate well with long-term outcomes as measured by VNPS or RMDI. In contrast, the 2-week VNPS had a correlation with the long-term response at 2 months for VNPS and RMDI outcomes. Univariate logistic regression also defined that the 2-week VNPS would be a predictor of patients achieving a $>50 \%$ improvement at 2 months (Table 4). To be clear, the immediate postprocedure pain response did not predict outcomes, but the 2-week VNPS was a significant predictor of patients who would maintain a $>50 \%$ improvement. The results of this analysis also show that the higher the 2-week pain score, the less likely the patient is to achieve $>50 \%$ relief at 2 months. The odds ratio of 0.789 indicates that on average, a patient would have 2.6 times better odds of achieving the $50 \%$ improvement than a patient with a 4 -point higher baseline VNPS.

As with the VNPS, the 2-week RMDI score also was a significant predictor of patients who would have a sustained 50\% improvement in RMDI at 2 months. Collectively, the data show that clinicians ular pain through its direct effect on the sensory nerve fibers, and studies have shown that it can decrease leukocyte response, ${ }^{9,10}$ it likely has minimal direct effect on the inflammatory cascade thought to be partially responsible for pain generation in radicular pain. The etiology of radicular pain is probably multifactorial and involves multiple mechanisms that cannot be immediately ameliorated with anesthetics or steroids.

Though Mixter and Barr ${ }^{11}$ demonstrated that sciatica was associated with disk herniation in 1934, recent research has demonstrated that radicular pain is not only caused by mechanical injury but also by chemical inflammatory factors. ${ }^{12-17}$ These findings are substantiated by clinical findings such as asymptomatic prominent disk protrusions, radicular pain without mass effect on a nerve, and trials demonstrating improvement in pain without surgical decompression. ${ }^{18-21}$

One significant cause of radicular pain is the inflammatory response that is present at the treatment level. Indeed, the antiinflammatory properties of steroids are a major rationale for these injections. ${ }^{12,22}$ There are many mediators that can generate an inflammatory response. High levels of phospholipase A2 are found in the intervertebral disk, and elevated levels of PLA2 have been found in tissues from human radiculopathy patients ${ }^{17}$ and animal models. ${ }^{16}$ PLA2 initiates the arachidonic acid cascade that leads to the production of prostaglandins, thromboxanes, and leukotrienes, all inflammatory mediators. ${ }^{12,22}$ Inflammatory mediators, in turn, cause a range of effects including recruitment of immune cells (leukocytes, lymphocytes, macrophages, platelets, and mast cells) and the subsequent activation of these cells, edema through effects on the vasculature, and production of inflammatory cytokines among oth-

Table 4: Univariate logistic regression models using pain and RMDI scores as predictors of later response

\begin{tabular}{llcc}
\multicolumn{1}{c}{ Outcome } & \multicolumn{1}{c}{ Predictor } & Odds Ratio & $\begin{array}{c}\text { 95\% Confidence } \\
\text { Interval }\end{array}$ \\
\hline$>50 \%$ Improvement VNPS at 2 weeks & Immediate VNPS & 0.848 & $(0.710,1.012)$ \\
$>50 \%$ Improvement VNPS at 2 months & Immediate VNPS & 0.944 & $(0.777,1.147)$ \\
$>50 \%$ Improvement VNPS at 2 months & 2-Week VNPS & 0.789 & $(0.662,0.941)$ \\
$>50 \%$ Improvement RMDI at 2 weeks & Immediate VNPS & 0.863 & .067 \\
$>50 \%$ Improvement RMDI at 2 months & Immediate VNPS & 0.973 & $.0 .701,1.062)$ \\
$>50 \%$ Improvement RMDI at 2 months & 2-Week RMDI & 0.853 & $(0.799,1.185)$ \\
\end{tabular}


ers. The inflammatory response can cause additional tissue injury, including Wallerian degeneration. This cascade also can increase pain through hypersensitization of the neural roots to stimuli that may not normally be painful. ${ }^{12,22}$

The transforaminal injection of steroids can be effective by interacting with the inflammatory response at several points. A primary target of the steroid is the inflammatory cascade, particularly the effects of phospholipase A2 and the lipoxygenase pathway, thus reducing the downstream production of inflammatory mediators. Steroids also can reduce recruitment of leukocytes, capillary permeability, and edema; reduce the release of damaging mediators from activated leukocytes; and directly reduce the activity of pain pathways. Interruption of these cascades occurs over a period of days; thus, the effect of steroids is not immediate but becomes manifest over the days and weeks after treatment.

Given the complexity of the physical and inflammatory mechanisms that can be involved in causing radicular pain, it should not be surprising that a time point that allows the steroid effect to become manifest would be a better predictor of later outcomes for the patient. In this study, the 2-week pain and RMDI scores significantly correlated with the latter 2-month outcome, whereas the immediate postprocedure pain scores did not. The statistical analysis also shows that the lower the pain and RMDI scores at this 2-week time point, the higher the likelihood that this positive effect will persist at the 2-month time point. Though we as clinicians would like to offer patients hope on the basis of the anesthetic effect at the time of a procedure, ultimately we must counsel them that the true predictor of long-term relief will come once the steroids have been given time to have a localized effect on the nerves and surrounding tissues.

This study is limited by several factors. Study participants were referred for injection by use of a synthesis of multiple criteria including history and physical examination, imaging, and electromyography. This inevitably leads to heterogeneity in a population. Duration of pain and level of radiculopathy were not stratified. Further studies may be necessary to define whether a more limited patient cohort would affect outcomes. Loss to follow-up was expected; however, it may be a potential source of bias, as previously reported. ${ }^{5}$ Baseline VNPS and RMDI were not significantly different between the groups of patients with and without 2 -week data. Further analysis of those lost to follow-up revealed that 2-week outcome was not associated with 2-month loss to follow-up, but baseline VNPS/RMDI was higher in those lost to follow-up at 2 months. This may conservatively bias our reported proportions of favorable response because the group of 2-month $50 \%$ responders had higher baseline RMDI than 50\% nonresponders; therefore no missing data adjustments are made. Finally, all of these patients underwent CT-guided procedures. These results are probably transferable to fluoroscopic-guided cervical transforaminal epidural injections, but this cannot be ensured without a definitive study in that image-guided group.

\section{CONCLUSIONS}

Pain scores obtained immediately after completion of a single CT-guided CTESI do not predict the long-term efficacy of this procedure. However, patient response at 2 weeks does correlate with the long-term efficacy of these injections as measured by the VNPS and the RMDI.
Disclosures: Kent Thielen—UNRELATED: Royalties: Nevro Inc; Stock/Stock Options: Nevro Inc.

\section{REFERENCES}

1. Castagnera L, Maurette P, Pointillart V, et al. Long-term results of cervical epidural steroid injection with and without morphine in chronic cervical radicular pain. Pain 1994;58:239-43

2. Kolstad F, Leivseth G, Nygaard OP. Transforaminal steroid injections in the treatment of cervical radiculopathy: a prospective outcome study. Acta Neurochir (Wien) 2005;147:1065-70

3. Lin EL, Lieu V, Halevi L, et al. Cervical epidural steroid injections for symptomatic disc herniations. J Spinal Disord Tech 2006;19:183-86

4. Slipman CW, Lipetz JS, Jackson HB, et al. Therapeutic selective nerve root block in the nonsurgical treatment of atraumatic cervical spondylotic radicular pain: a retrospective analysis with independent clinical review. Arch Phys Med Rehabil 2000;81:741-46

5. Wald JT, Maus TP, Geske JR, et al. Safety and efficacy of CT-guided transforaminal cervical epidural steroid injections using a posterior approach. AJNR Am J Neuroradiol 2012;33:415-19

6. Roland M, Morris R. A study of the natural history of back pain, part I: development of a reliable and sensitive measure of disability in low-back pain. Spine 1983;8:141-44

7. Cohen SP, Hurley RW. The ability of diagnostic spinal injections to predict surgical outcomes. Anesth Analg 2007;105:1756-75

8. Cyteval C, Thomas E, Decoux E, et al. Cervical radiculopathy: open study on percutaneous periradicular foraminal steroid infiltration performed under CT control in 30 patients. AJNR Am J Neuroradiol 2004;25:441-45

9. MacGregor RR, Thorner RE, Wright DM. Lidocaine inhibits granulocyte adherence and prevents granulocyte delivery to inflammatory sites. Blood 1980;56:203-09

10. Cullen BF, Haschke RH. Local anesthetic inhibition of phagocytosis and metabolism of human leukocytes. Anesthesiology 1974;40:142-46

11. Mixter WJ, Barr JS. Rupture of the intervertebral disc with involvement of the spinal canal. $N$ Engl J Med 1934;211:210-14

12. Baqai $A, B a l R$. The mechanism of action and side effects of epidural steroids. Tech Reg Anesth Pain Manag 2009;13:205-11

13. Kawakami M, Matsumoto T, Tamaki T. Roles of thromboxane A2 and leukotriene $B 4$ in radicular pain induced by herniated nucleus pulposus. J Orthop Res 2001;19:472-77

14. Kobayashi S, Baba H, Uchida K, et al. Effect of mechanical compression on the lumbar nerve root: localization and changes of intraradicular inflammatory cytokines, nitric oxide, and cyclooxygenase. Spine 2005;30:1699-705

15. Kobayashi S, Yoshizawa H, Yamada S. Pathology of lumbar nerve root compression, part 1: intraradicular inflammatory changes induced by mechanical compression. J Orthop Res 2004;22:170-79

16. Lee HM, Weinstein JN, Meller ST, et al. The role of steroids and their effects on phospholipase A2. An animal model of radiculopathy. Spine 1998;23:1191-96

17. Saal JS, Franson RC, Dobrow R, et al. High levels of inflammatory phospholipase A2 activity in lumbar disc herniations. Spine 1990;15:674-78

18. Atlas SJ, Keller RB, Chang Y, et al. Surgical and nonsurgical management of sciatica secondary to a lumbar disc herniation: five-year outcomes from the Maine Lumbar Spine Study. Spine 2001;26:1179-87

19. Ohnmeiss DD, Vanharanta H, Ekholm J. Degree of disc disruption and lower extremity pain. Spine 1997;22:1600-05

20. Vucetic N, de Bri E, Svensson O. Clinical history in lumbar disc herniation: a prospective study in $\mathbf{1 6 0}$ patients. Acta Orthop Scand 1997;68:116-20

21. Weber H. Lumbar disc herniation: a controlled, prospective study with ten years of observation. Spine 1983;8:131-40

22. McLain RF, Kapural L, Mekhail NA. Epidural steroid therapy for back and leg pain: mechanisms of action and efficacy. Spine $J$ 2005;5:191-201 